Series A

\author{
I. MATHEMATICA
}

395

\title{
CHARACTERIZATION \\ OF QUASICONFORMAL ARCS
}

BY

SEPPO RICKMAN

H EL S IN I 1966

S U O M A L I N EN TIEDEAKATEMIA

doi:10.5186/aasfm.1967.395 
Communicated 11 November 1966 by OlLi Lehto and K. I. Virtanen 


\section{Preface}

I am deeply indebted to Professor Olli Lehto and Professor K. I. Virtanen for suggesting this subject and for their kind interest and valuable advice.

I also wish to acknowledge my gratitude for a State grant for young scientists and to thank the Finnish Academy of Sciences for financial support.

Helsinki, November 1966

Seppo Rickman 


\section{Contents}

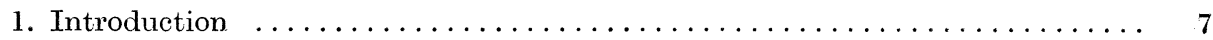

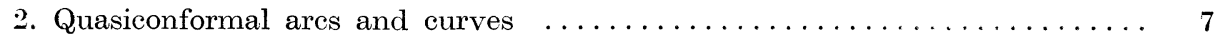

3. Bounded distortion of Jordan ares and curves $\ldots \ldots \ldots \ldots \ldots \ldots \ldots \ldots . \ldots$

4. Characterization of closed quasiconformal ares $\ldots \ldots \ldots \ldots \ldots \ldots \ldots \ldots, 12$

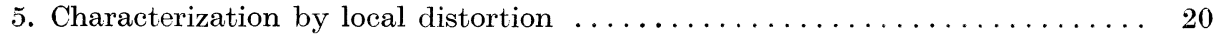

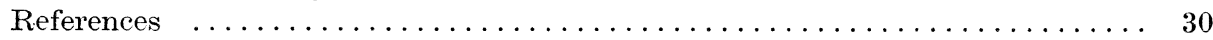




\section{Introduction}

The quasiconformality of Jordan arcs and curves in the sense of Lehto and Virtanen [5] is closely related to the possibility of extending quasiconformal mappings of plane domains. The extension problem was first treated by Tienari [8], and some of the results were generalized by Väisälä [9]. However, the conditions for quasiconformality of ares and curves contained in [8] are rather implicit; cf. also Pfluger [7]. Accordingly, it has been natural to ask for more direct characterizations. A solution of this kind was established for Jordan curves by Ablfors [2]. He showed that quasiconformal curves can be characterized by a surprisingly simple metrical condition; in this paper we say that a Jordan arc or curve has bounded distortion if it satisfies such a condition. The main results in [2], [8], [9], and this work are essentially based on the important paper [3] by Beurling and Ahlfors about the boundary correspondence under quasiconformal mappings.

It has been an open question whether the quasiconformality of a Jordan arc can also be characterized by the distortion of the arc. The answer is affirmative for closed arcs, which is proved in Section 4. In Section 5 it is first shown that, in the case of open ares, bounded distortion implies quasiconformality but not conversely. A characterization of quasiconformality for open ares is obtained in terms of a measure of local distortion. With the help of the local distortion we also give bounds for the maximal dilatation which appears in the definition of quasiconformality of Jordan arcs and curves. In order to obtain a best possible lower bound we have used recent results concerning topological angles introduced by Agard and Gehring [1] in connection with quasiconformal mappings.

\section{Quasiconformal ares and curves}

2.1. We consider sense-preserving homeomorphisms $f: G \rightarrow G^{\prime}$, where $G$ and $G^{\prime}$ are domains in the extended plane. The maximal dilatation of such a mapping $f$ is denoted by $K(f)$. The definition in terms of quadrilaterals may be found in [5], p. 17. If $K(f)<\infty$, the mapping $f$ is said to be quasiconformal, and if $K(f) \leq K<\infty, f$ is called $K$ quasiconformal. 
2.2. Let $\gamma$ be a Jordan $\operatorname{arc}^{1}$ ) or curve in the extended plane. We say that $\gamma$ is $K$-quasiconformal if there is a $K$-quasiconformal mapping $f$ of a domain $G \supset \gamma$ such that $f(\gamma)$ is a line segment or a circle respectively. If $\gamma$ is $K$-quasiconformal for some $K$, it is called quasiconformal. We denote

$$
K(\gamma)=\inf _{f \in F(\gamma)} K(f),
$$

where $F(\gamma)$ is the set of all sense-preserving plane homeomorphisms $f$ of domains $G \supset \gamma$ such that $f(\gamma)$ is a line segment or a circle respectively. Then $\gamma$ is quasiconformal if and only if $K(\gamma)$ is finite, and if $\gamma$ is $K$ quasiconformal, the inequality $K(\gamma) \leq K$ holds.

It follows from an extension theorem ([5], Satz II.8.1) that if $\gamma$ is closed, the domain $G$ in the definition of quasiconformality of $\gamma$ can be chosen to be the whole plane. An immediate consequence of this is the result that a closed Jordan arc is quasiconformal if and only if it is a subarc of a quasiconformal curve (see [5], p. 102).

2.3. Open quasiconformal arcs and quasiconformal curves are related to the extension of quasiconformal mappings according to the following lemma.

Lemma 1. Let $G$ and $G^{\prime}$ be domains with $\gamma$ and $\gamma^{\prime}$ as open free boundary $\operatorname{arcs}^{2}$ ) or free boundary curves respectively. If $\gamma$ and $\gamma^{\prime}$ are $K$ - and $K^{\prime}$-quasiconformal respectively and if $f: G \rightarrow G^{\prime}$ is a $K_{0}$-quasiconformal mapping with $\gamma$ and $\gamma^{\prime}$ corresponding to each other, then $f$ can be extended to a $K^{2} K_{0} K^{\prime 2}$-quasiconformal mapping of a domain containing $G \cup \gamma$.

This lemma is a slightly modified form of Satz II.8.2 in [5], and for the proof we refer to [5].

2.4. Local quasiconformality. We also define a concept of local quasiconformality in the following manner (cf. [9]). A Jordan arc or curve $\gamma$ is called (K-)quasiconformal at the point $z \in \gamma$, if $z$ belongs to a $(K-)$ quasiconformal subarc $\gamma_{z} \subset \gamma$ open in $\gamma$. If $z$ is a point of $\gamma$, we denote

$$
K(\gamma, z)=\inf _{\gamma_{z}} K\left(\gamma_{z}\right)
$$

where the infimum is taken over all subarcs $\gamma_{z} \subset \gamma$ containing $z$ and open in $\gamma$. Then $\gamma$ is quasiconformal at $z \in \gamma$ if and only if $K(\gamma, z)$ is finite. If $\gamma$ is $K$-quasiconformal at $z \in \gamma$, then $K(\gamma, z) \leq K$.

$\left.{ }^{1}\right)$ A Jordan arc is here defined to be homeomorphic to a line segment. The closure of an open Jordan arc is then not necessarily a Jordan are.

$\left.{ }^{2}\right)$ We define a free boundary arc of a domain as in [5], p. 12. 
Clearly quasiconformality of $\gamma$ implies quasiconformality at every point $z \in \gamma$. It has also been proved that a Jordan curve is quasiconformal if it is quasiconformal at all of its points ([8], Satz 12, and [5], p. 108). We give this and the corresponding result for closed Jordan arcs in the corollary of Theorem 4.

\section{Bounded distortion of Jordan ares and curves}

3.1. We introduce now the condition of Ahlfors appearing in [2]. Let $\left|z_{1}, z_{2}, z_{3}, z_{4}\right|$ denote the absolute value of the cross ratio of the sequence $z_{1}, z_{2}, z_{3}, z_{4}$. For finite distinct points $z_{1}, z_{2}, z_{3}, z_{1}$ we have then

$$
\left|z_{1}, z_{2}, z_{3}, z_{4}\right|=\frac{z_{1}-z_{3}|| z_{2}-z_{4} \mid}{\left|z_{1}-z_{4}\right| z_{2}-z_{3} \mid} .
$$

We say that a Jordan arc or curve $\gamma$ has bounded distortion if there exists a number $c, 1 \leq c<\infty$, such that

$$
\left|z_{1}, z_{4}, z_{2}, z_{3}+z_{1}, z_{2}, z_{4}, z_{3}\right| \leq c
$$

for all sequences $z_{1}, z_{2}, z_{3}, z_{4}$ of successive points of $\gamma$. We call $c(\gamma)$, the supremum of the left side of (1), the distortion of $\gamma$. This is clearly invariant under linear transformations. The distortion $c(\gamma)$ equals 1 if and only if $\gamma$ is contained in a line or a circle.

In [4] a Jordan curve lying in the three dimensional space and satisfying (1) is called a c-circle. For other forms, equivalent to (1) in case of Jordan curves, we refer to [2] and [5], p. 104, 107.

Remark. The condition for Jordan ares given in [5], p. 107 (beschränkte Schwenkung), is equivalent to (1) only if the arc is closed.

3.2. If $\gamma$ is a Jordan arc, and $w_{1}$ and $w_{2}$ are two points of $\gamma$, we denote throughout this paper by $\gamma\left(u_{1}, w_{2}\right)$ the open subarc of $\gamma$ which has the end-points $u_{1}$ and $w_{2}$. The euclidean diameter of a set $A$ is denoted by $d(A)$.

For later applications we consider a closed Jordan arc $\gamma$ with $\infty$ as one end-point. Fixing $z_{1}=\infty$ in (1), we obtain the simple condition

$$
\left|z_{1}-z_{2}\right|+\left|z_{2}-z_{3}\right| \leq c\left|z_{1}-z_{3}\right|
$$

for successive finite points $z_{1}, z_{2}, z_{3}$ of $\gamma$. With the above notation $\gamma$ also satisfies the condition

$$
d\left(\gamma\left(z_{1}, z_{2}\right)\right) \leq c\left|z_{1}-z_{2}\right|
$$

for finite points $z_{1}, z_{2} \in \gamma$. 
3.3. The remarkable result that a Jordan curve is quasiconformal if and only if it has bounded distortion was first proved by Ahlfors in [2]. For a slightly different proof, see [5], Satz II.8.6. In this paper we have the result as a corollary of Theorem 1 .

If a Jordan arc or curve $\gamma$ has bounded distortion, it follows directly from the definition that every subarc of $\gamma$ has bounded distortion. Since a closed quasiconformal are is a subarc of a quasiconformal curve, and quasiconformal curves have bounded distortion, we have the result that closed quasiconformal arcs have bounded distortion. We shall show in the next section that the converse also holds.

3.4. Local distortion. In analogy with the concept of local quasiconformality we now introduce a measure of local distortion in the following manner. Let $\gamma$ be a Jordan arc or curve and let $z$ be a point of $\gamma$. We call

$$
c(\gamma, z)=\inf _{\gamma_{z} z_{2} \in \sup _{\gamma_{z}\left(z_{1}, z_{3}\right)}} \frac{k\left(z_{1}, z_{2}\right)+k\left(z_{2}, z_{3}\right)}{k\left(z_{1}, z_{3}\right)},
$$

where $k$ is the spherical metric, and the infimum is taken over all subarcs $\gamma_{z} \subset \gamma$ containing $z$ and open in $\gamma$, the distortion of $\gamma$ at the point $z$. If $z \neq \infty$, the spherical metric can here be replaced by the euclidean. We call further

$$
c_{0}(\gamma)=\sup _{z \in \gamma} c(\gamma, z)
$$

the maximal local distortion of $\gamma$ and say that $\gamma$ has bounded local distortion if $c_{0}(\gamma)$ is finite.

We prefer to choose $c(\gamma, z)$ as a measure for local distortion rather than

$$
c_{*}(\gamma, z)=\inf _{\gamma_{z}} c\left(\gamma_{z}\right)
$$

where $\gamma_{z}$ varies as above. There are two reasons for this. For one thing, $c(\gamma, z)$ is simpler in calculation than $c_{*}(\gamma, z)$. Secondly, a sharp lower bound for $K(\gamma)$ is easily obtained in Section 5 in terms of $c_{0}(\gamma)$.

In the following sense $c(\gamma, z)$ is equivalent to $c_{*}(\gamma, z)$ : The inequality $c(\gamma, z) \leq c_{*}(\gamma, z)$ holds, and there is an upper bound for $c_{*}(\gamma, z)$ depending only on $c(\gamma, z)$. To show that $c(\gamma, z) \leq c_{*}(\gamma, z)$ is true, suppose first that $z \neq \infty$, take $\varepsilon>0$, and let $\gamma_{z} \nexists \infty$ be a subarc of $\gamma$ open in $\gamma$ and containing the point $z$. Given $z_{4} \in \gamma_{z}, z_{4} \neq z$, we choose a $\varrho>0$ such that $(r-\varrho) /(r+\varrho)>1-\varepsilon$, where $r=\left|z_{4}-z\right|$. Assume $a<c(\gamma, z)$. There exist points $z_{i} \in \gamma_{z}, i=1,2,3$, such that $\left|z_{i}-z\right|<\varrho$, that $z_{1}, z_{2}, z_{3}, z_{4}$ are successive, and that

$$
\lambda\left(z_{1}, z_{2}, z_{3}\right)=\frac{\left|z_{1}-z_{2}\right|+\left|z_{2}-z_{3}\right|}{\left|z_{1}-z_{3}\right|}>a .
$$


Then $\left|z_{3}-z_{4}\right|,\left|z_{1}-z_{4}\right|>r-\varrho$ and $\left|z_{2}-z_{4}\right|<r+\varrho$. Hence

$$
\begin{gathered}
\left|z_{1}, z_{4}, z_{2}, z_{3}\right|+\left|z_{1}, z_{2}, z_{4}, z_{3}\right|=\frac{\left|z_{1}-z_{2}\right|\left|z_{3}-z_{4}\right|+\left|z_{1}-z_{4}\right|\left|z_{2}-z_{3}\right|}{\left|z_{1}-z_{3}\right|\left|z_{2}-z_{4}\right|} \\
>(1-\varepsilon) \lambda\left(z_{3}, z_{2}, z_{3}\right)>(1-\varepsilon) a .
\end{gathered}
$$

Accordingly, the assertion is proved in case $z \neq \infty$. It is easy to show that $c(\gamma, z)$ is invariant under linear transformations. Since this holds trivially for $c_{*}(\gamma, z)$, we conclude that the asserted inequality is true also if $z=\infty$. An upper bound for $c_{*}(\gamma, z)$ in terms of $c(\gamma, z)$ is obtained by elementary estimates.

The connection between local distortion and quasiconformality is discussed in Section 5.

If $\gamma$ is a closed Jordan arc or a Jordan curve, then $\gamma$ has bounded local distortion if and only if the distortion of $\gamma$ at every point $z \in \gamma$ is finite. In this case, also the property of having bounded distortion in the large can be characterized locally:

Lemma 2. A closed Jordan arc or a Jordan curve $\gamma$ has bounded distortion if and only if $c(\gamma, z)$ is finite at every point $z \in \gamma$.

The proof of this lemma is included in the proof of Satz II.8.7 in [5] in the case where $\gamma$ is a Jordan curve. The proof for closed Jordan arcs is similar (cf. the remark on p. 9).

Lemma 2 does not hold for open arcs. The open Jordan arc

$$
\gamma=\left\{(x, y) \mid y=\sin \frac{1}{x}, 0<x<1\right\}
$$

has bounded local distortion, because $c(\gamma, z)=1$ at every point $z \in \gamma$. However, $\gamma$ does not have bounded distortion.

3.5. Connection with topological angles. The inner measure of a topological angle, defined in [1], is closely related to the local distortion. A pair $\left(\gamma_{1}, \gamma_{2}\right)$ of two closed Jordan arcs $\gamma_{1}$ and $\gamma_{2}$ forms a topological angle at a point $z_{0} \neq \infty$ if both $\gamma_{1}$ and $\gamma_{2}$ have $z_{0}$ as an end-point and if $z_{0}$ is the only point $\gamma_{1}$ and $\gamma_{2}$ have in common. The inner measure $A\left(\gamma_{1}, \gamma_{2}\right)$ of this topological angle $\left(\gamma_{1}, \gamma_{2}\right)$ is defined as follows:

$$
A\left(\gamma_{1}, \gamma_{2}\right)=\lim _{\substack{z_{1}, z_{2} \rightarrow z_{0} \\ z_{i} \in \gamma_{i}}} 2 \arcsin \left(\lambda\left(z_{1}, z_{0}, z_{2}\right)\right)^{-1},
$$

where $\lambda$ is defined by (4).

Let now $\left(\gamma_{1}, \gamma_{2}\right)$ be a topological angle at $z_{0}$. Then $\gamma=\gamma_{1} \cup \gamma_{2}$ is a Jordan arc, and the distortion $c\left(\gamma, z_{0}\right)$ of $\gamma$ at the point $z_{0}$ is defined. It follows directly from the definitions that the following inequality holds: 


$$
A\left(\gamma_{1}, \gamma_{2}\right) \geq 2 \arcsin \frac{1}{c\left(\gamma, z_{0}\right)} .
$$

If $\gamma_{1}$ and $\gamma_{2}$ are line segments, there is equality in (6). It is also easy to see by examples that there exist topological angles $\left(\gamma_{1}, \gamma_{2}\right)$ with $A\left(\gamma_{1}, \gamma_{2}\right)$ greater than the right side of (6).

\section{Characterization of closed quasiconformal ares}

4.1. The main purpose of this section is to prove Theorem 1, which shows that the quasiconformality of a closed Jordan arc can be characterized by the distortion of the arc. Before starting the proof we introduce some notation.

If not otherwise stated, let $\gamma$ be in this section a closed Jordan arc. Let $\omega$ map $C_{\gamma}$, the complement of $\gamma$, conformally onto the upper half plane $H$. The mapping (') induces a natural one-to-one correspondence between $\Gamma$, the set of boundary elements of $C_{\gamma}$, and the boundary $\partial H$ of $H$. We denote this natural extension of $\omega$, which is a mapping of $C_{\gamma} \cup \Gamma$ onto the closure $\bar{H}$ of $H$, by the same symbol $\omega$.

Let now $\zeta_{1}, \zeta_{2}, \zeta_{3}, \zeta_{4}$ be four distinct points of $\Gamma$ such that the sequence $\omega\left(\zeta_{1}\right), \ldots, \omega\left(\zeta_{4}\right)$ has positive orientation with respect to $H$. We call the pair consisting of the domain $C_{y}$ and the sequence $\zeta_{1}, \ldots, \rightarrow_{-1}$ an arcquadrilateral and denote it by $C_{\gamma}\left(\zeta_{1}, \ldots, \zeta_{4}\right)$. This is a natural modification of the concept of quadrilateral corresponding to Jordan domains (see e.g. [5], p. 15). The modulus $M\left(C_{\gamma}\left(\zeta_{1}, \ldots, \zeta_{4}\right)\right)$ of $C_{.}\left(\varsigma_{-1}, \ldots, \varsigma_{4}\right)$ is defined to be the modulus of the quadrilateral $H\left(\omega\left(\varsigma_{-1}\right), \ldots, \omega\left(\varsigma_{4}\right)\right)$. It is clear that this definition of the modulus of an arc-quadrilateral does not depend on the choice of the conformal mapping $a$. The $a$ - and $b$ sides of $C_{\gamma}\left(\zeta_{1}, \ldots, \zeta_{4}\right)$, denoted $\varsigma_{1} \zeta_{-2}, \zeta_{3} \zeta_{4}$ and $\zeta_{2} \zeta_{3}, \zeta_{1} \varsigma_{1}$ respectively, are defined to be the subsets of $\Gamma$ corresponding to the $a$ - and $b$-sides of $H\left(\omega\left(\zeta_{1}\right), \ldots, \omega\left(\zeta_{4}\right)\right)$ ([5], p. 15) under the mapping (") .

An arc joining points $\zeta$ and $\zeta$ of $\Gamma$ in $C$ is an open Jordan arc $\alpha \subset C_{\gamma}$ such that $\overline{\omega(\alpha)}-\omega(\alpha)=\left\{\omega\left(\begin{array}{c}\infty \\ \varnothing\end{array}\right), \omega\left(\begin{array}{c}\infty \\ =\end{array}\right)\right\}$. If $A$ is the family of arcs joining points of opposite $a$-sides of $\left.C_{\gamma(-1}, \ldots, s_{4}\right)$ in $C_{\gamma}$, the modulus $M(A)$ of $A([5]$, p. 139$)$ is equal to $M\left(C_{2}\left(\zeta_{1}, \ldots, \zeta_{4}\right)\right)$.

Let $p$ and $q$ be the end-points of $\gamma$ in the following. We fix the mapping $\omega$ so that the points $p, 0$ and $q, \infty$ correspond to each other on the boundary. Let $P$ be the natural projection of $\Gamma$ onto $\gamma$. If $z \in \gamma^{\prime}$, we denote by $z^{-}$and $z^{+}$the points of $\Gamma$ for which $P\left(z^{-}\right)=P\left(z^{+}\right)=z$, $\omega\left(z^{-}\right)$is non-positive, and $\omega\left(z^{+}\right)$is non-negative. Then $p^{-}=p^{+}$and 
$q^{-}=q^{+}$, and there is no confusion if these are simply denoted by $p$ and $q$ respectively. We also fix a natural order on the points of $\gamma$ so that $p<q$.

4.2. We are now ready to state the lemmas which are needed in the proof of Theorem 1 .

Lemma 3. Let $\gamma$ have bounded distortion and suppose that the end-point $q$ is $\infty$. Then there exists a constant $k_{1}<\infty$ depending only on $c=c(\gamma)$ such that the condition

$$
\frac{1}{k_{1}} \leq M\left(C_{\gamma}\left(z_{1}^{+}, z_{2}^{+}, z_{3}^{+}, \infty\right)\right) \leq k_{1}
$$

holds for finite points $z_{1}, z_{2}, z_{3} \in \gamma$ satisfying $z_{1}<z_{2}<z_{3}$ and

$$
M\left(C_{\gamma}\left(\infty, z_{3}^{-}, z_{2}^{-}, z_{1}^{-}\right)\right)=1 \text {. }
$$

Proof. Let $z_{1}, z_{2}$, and $z_{3}$ satisfy the conditions in the lemma. We show first that

$$
\frac{d_{0}}{8 c^{2} e^{2 \cdot \tau}} \leq d_{a} \leq 2 c d_{0} e^{2 . \tau}
$$

holds, where $d_{a}$ is the euclidean distance between the arcs $\gamma\left(z_{1}, z_{2}\right)$ and $\gamma\left(z_{3}, \infty\right)$, and $d_{0}=\left|z_{1}-z_{2}\right|$.

To prove the left inequality of (7), suppose

$$
\frac{d_{0}}{8 c^{2} e^{2 \cdot \tau}}>d_{a}
$$

and set $r_{1}=c d_{a}$ and $r_{2}=2 c d_{a} e^{2 \cdot \tau}$. It follows from (3) that there exists a point $z_{0}$ such that the disk $\left\{z \mid z-z_{0} \leq r_{1}\right\}$ contains the arc $\gamma\left(z_{2}, z_{3}\right)$. Let $\varrho_{1}$ be a function defined as follows:

$$
\varrho_{1}(z)=\left\{\begin{array}{l}
\frac{1}{\log \frac{r_{2}}{r_{1}}} \cdot \frac{1}{\left|z-z_{0}\right|}, \text { if } r_{1} \leq\left|z-z_{0}\right| \leq r_{2} \\
0 \text { elsewhere } .
\end{array}\right.
$$

Let $\beta^{-}$be an arc joining points $\zeta \in z_{3}^{-} z_{2}^{-}$and $\zeta^{\prime} \in z_{1}^{-} \infty$ in $C_{\gamma}$. Denote $z=P(\zeta)$ and $z^{\prime}=P\left(\zeta^{\prime}\right)$. Then $z \in \gamma\left(z_{2}, z_{3}\right)$. If $z^{\prime} \in \overline{\gamma\left(p, z_{1}\right)}$, it follows from (3) that $d_{0} \leq c\left|z-z^{\prime}\right|$, and so $d\left(\beta^{-}\right) \geq d_{0} / c$. Assume $z^{\prime} \notin \overline{\gamma\left(p, z_{1}\right)}$, and let $D^{\prime}$ be the component of $H-\omega\left(\beta^{-}\right)$which has 0 as a boundary point. Denote $D=\omega^{-1}\left(D^{\prime}\right)$, and let $G$ be the domain which is bounded by the Jordan curve $\overline{\beta^{-}} \cup \gamma\left(z, z^{\prime}\right)$ and which contains the point $p$. Then $\gamma(p, w) \subset G$ and $w \in \hat{c} G$, where $w=\min \left\{z, z^{\prime}\right\}$. The boundary of $D$ 
consists of $\overline{\gamma(p, w)}$ and the Jordan curve $\partial G$. Hence it follows that $D=G-\overline{\gamma(p, w)}$, because $p \in G$. On the other hand, $z_{1}, z_{2} \in \partial D$, and so $z_{1}, z_{2} \in \bar{G}$. Since $\infty \notin G$, we have $d(G)=d(\partial G)$ and thus $d_{0} \leq d(\partial G)$. The diameter $d(\partial G)$ is at most $d\left(\beta^{-}\right)+d\left(\gamma\left(z, z^{\prime}\right)\right)$, and so we have

$$
d_{0} \leq d\left(\beta^{-}\right)+d\left(\gamma\left(z, z^{\prime}\right)\right) .
$$

If $d\left(\beta^{-}\right)<d_{\mathbf{0}} /(2 c) \leq d_{\mathbf{0}} / 2$, it follows from (8) that $d\left(\gamma\left(z, z^{\prime}\right)\right)>d_{\mathbf{0}} / 2$. But from $\left|z-z^{\prime}\right| \leq d\left(\beta^{-}\right)<d_{0} /(2 c)$ we obtain then $d\left(\gamma\left(z, z^{\prime}\right)\right)>$ c $\left|z-z^{\prime}\right|$, which is in contradiction with (3), and so in any case

$$
d\left(\beta^{-}\right) \geq \frac{d_{0}}{2 c}
$$

Hence, in view of our assumption,

$$
d\left(\beta^{-}\right)>4 c d_{a} e^{2 \pi}=2 r_{2} .
$$

From this and from $\left|z-z_{0}\right| \leq r_{1}$ it follows that there exists a point $z^{\prime \prime} \in \beta^{-}$such that $\left|z^{\prime \prime}-z_{0}\right|>r_{2}$. The $\varrho_{1}$-length of $\beta^{-}$is therefore at least 1 and we obtain the estimate ([5], p. 139)

$$
\begin{aligned}
\frac{1}{M\left(C_{\gamma}\left(\infty, \tilde{\sim}_{3}^{-}, z_{2}^{-}, z_{1}^{-}\right)\right)} & =M\left(C_{\gamma}\left(z_{3}^{-}, z_{2}^{-}, z_{1}^{-}, \infty\right)\right) \leq \iint \varrho_{1}^{2} d \sigma \\
& =\frac{2 \pi}{\left(\log \frac{r_{2}}{r_{1}}\right)^{2}} \int_{r_{1}}^{r_{2}} \frac{d r}{r}<1,
\end{aligned}
$$

which contradicts the assumption of the lemma.

To prove the right side of (7), we observe as above that there exists a $z_{0}^{\prime}$ such that the arc $\gamma\left(z_{1}, z_{2}\right)$ is contained in the disk $\left\{z|| z-z_{0}^{\prime} \mid \leq c d_{0}\right\}$. If $2 c d_{0} e^{2 \pi}<d_{a}$, the arc $\gamma\left(z_{3}, \infty\right)$ lies outside a concentric disk with radius $2 c d_{0} e^{2 \pi}-2 c d_{0}$, and the $a$-sides of $C_{\gamma}\left(\infty, z_{3}^{-}, z_{2}^{-}, z_{1}^{-}\right)$as point sets are thus separated by a circular annulus whose radii have a ratio greater than $e^{2 \pi}$. The use of a similar $Q$-function as above yields the contradiction $M\left(C_{\gamma}\left(\infty, z_{3}^{-}, z_{2}^{-}, z_{1}^{-}\right)\right)<1$.

We now derive the upper bound for $M\left(C_{,}\left(z_{1}^{+}, z_{2}^{-}, z_{3}^{-}, \infty\right)\right)$ by defining

$$
\varrho_{2}(z)=\left\{\begin{array}{l}
\frac{8 c^{2} e^{2 \cdot \tau}}{d_{0}}, \text { if }\left|z-z_{0}^{\prime}\right| \leq c d_{0}+\frac{d_{0}}{8 c^{2} e^{2 . \tau}}, \\
0 \text { elsewhere },
\end{array}\right.
$$

where $z_{0}^{\prime}$ is as before. From the left inequality in (7) it follows that the $\varrho_{2}$-length of every arc joining the $a$-sides of $C_{\gamma}\left(z_{1}^{+}, z_{2}^{+}, z_{3}^{+}, \infty\right)$ in $C_{\gamma}$ is at least 1 . Hence we have the estimate 


$$
\begin{aligned}
M\left(C_{\gamma}\left(z_{1}^{+}, z_{2}^{+}, z_{3}^{+}, \infty\right)\right) & \leq \iint \varrho_{2}^{2} d \sigma=\pi\left(\frac{8 c^{2} e^{2 \pi}}{d_{0}}\right)^{2}\left(c d_{0}+\frac{d_{0}}{8 c^{2} e^{2 \pi}}\right)^{2} \\
& =\pi\left(8 c^{3} e^{2 \pi}+1\right)^{2}=k_{1} .
\end{aligned}
$$

Let now $\beta^{+}$be an arc joining the $b$-sides of $C_{\gamma}\left(z_{1}^{+}, z_{2}^{+}, z_{3}^{+}, \infty\right)$ in $C_{\gamma}$. As above we obtain the corresponding inequality (9) for $\beta^{+}$. Using the right side of (7) we have then for the euclidean length $l\left(\beta^{+}\right)$of $\beta^{+}$the inequality

$$
l\left(\beta^{+}\right) \geq \frac{d_{a}}{4 c^{2} e^{2 \cdot T}} .
$$

To obtain the lower bound, we define

$$
\varrho_{3}(z)=\left\{\begin{array}{l}
\frac{4 c^{2} e^{2 \pi}}{d_{a}}, \text { if }\left|z-z_{0}\right| \leq c d_{a}+\frac{d_{a}}{4 c^{2} e^{2 \pi}}, \\
0 \text { elsewhere },
\end{array}\right.
$$

where $z_{0}$ is as before. Since the arc $\gamma\left(z_{2}, z_{3}\right)$ is contained in the disk $\left\{z|| z-z_{0} \mid \leq c d_{a}\right\}$, it follows from (10) and the definition of $\varrho_{3}$ that every arc joining the $b$-sides of $C_{\gamma}\left(z_{1}^{+}, z_{2}^{+}, z_{3}^{+}, \infty\right)$ in $C_{\gamma}$ has a $\varrho_{3}$-length at least 1. Hence

$$
\frac{1}{M\left(C_{y}\left(z_{1}^{+}, z_{2}^{+}, z_{3}^{+}, \infty\right)\right)} \leq \pi\left(\frac{4 c^{2} e^{2 \pi}}{d_{a}}\right)^{2}\left(c d_{a}+\frac{d_{a}}{4 c^{2} e^{2 \pi}}\right)^{2}<k_{1} .
$$

The lemma is proved.

Lemma 4. Let $\gamma$ have bounded distortion and suppose that the end-point $q$ is $\infty$. Then there exists a constant $k_{2}<\infty$ depending only on $c=c(\gamma)$ such that for every point $w \in \gamma, w \neq p, \infty$, the condition

$$
\frac{1}{k_{2}} \leq M\left(C_{\gamma}\left(u^{-}, p, w^{-}, \infty\right)\right) \leq k_{2}
$$

holds.

Proof. Denote $d_{0}=|w-p|$, and let $\alpha$ be an arc joining points $; \in w^{-} p$ and $\zeta^{\prime} \in w^{+} \infty$ in $C \gamma$. As in the proof of Lemma 3, we obtain first the inequality

$$
d_{0} \leq d(\alpha)+d\left(\gamma\left(z, z^{\prime}\right)\right),
$$

where $z=P(\zeta)$ and $z^{\prime}=P\left(\zeta^{\prime}\right)$, and then by a similar argument for the euclidean length $l(\alpha)$ of $\alpha$ the inequality $l(\alpha) \geq d_{0} /(2 c)$. The arc $\gamma(p, w)$ is contained in a disk $\left\{z|| z-z_{0} \mid \leq c d_{0}\right\}$. If

$$
\varrho(z)=\left\{\begin{array}{l}
\frac{2 c}{d_{0}}, \text { if }\left|z-z_{0}\right| \leq c d_{0}+\frac{d_{0}}{2 c}, \\
0 \text { elsewhere, }
\end{array}\right.
$$


then the $\varrho$-length of every arc joining the $a$-sides of $C_{\gamma}\left(w^{-}, p, w^{+}, \infty\right)$ in $C \gamma$ is at least 1 , and

$$
M\left(C_{\gamma}\left(w^{-}, p, w^{+}, \infty\right)\right) \leq \pi\left(\frac{2 c}{d_{0}}\right)^{2}\left(c d_{0}+\frac{d_{0}}{2 c}\right)^{2}=\pi\left(2 c^{2}+1\right)^{2}=k_{2} .
$$

The lower bound $1 / k_{2}$ follows by symmetry.

Lemma 5. Let $\varphi$ be a strictly increasing function of the real line onto itself such that $\varphi(0)=0$, and denote

$$
\psi(x, t)=\frac{\varphi(x+t)-\varphi(x)}{\varphi(x)-\varphi(x-t)}
$$

for $t>0$. Let $\varphi$ satisfy the following conditions:

$1^{\circ}$. There exists a $\varrho_{1}<\infty$ such that

$$
\frac{1}{\varrho_{1}} \leq \psi(x, t) \leq \varrho_{1}
$$

for $t>0$, and $x-t \geq 0$ or $x+t \leq 0$.

$2^{\circ}$. There exists a $\varrho_{2}<\infty$ such that

$$
\frac{1}{\varrho_{2}} \leq \psi(0, t) \leq \varrho_{2}
$$

for $t>0$.

Then there exists a $\varrho<\infty$ depending only on $\varrho_{1}$ and $\varrho_{2}$ such that

$$
\frac{1}{\varrho} \leq \psi(x, t) \leq \varrho
$$

for all $x$ and for $t>0$, that is to say, $\varphi$ is Q-quasisymmetric.

Proof. Assume that $x, t>0$ and $x-t<0$. We consider first the case $x \geq t / 2$. Then we have by $2^{\circ}$

$$
\varphi(x)-\varphi(x-t) \leq \varphi(x)-\varphi(-x) \leq\left(1+\varrho_{2}\right) \varphi(x)
$$

and by $1^{\circ}$

$$
\varphi(x+t)-\varphi(x)>\varphi(2 x)-\varphi(x) \geq \frac{1}{\varrho_{1}} \varphi(x) .
$$

This implies the lower bound $1 /\left(\varrho_{1}\left(\varrho_{2}+1\right)\right)$ for $\psi(x, t)$. In order to obtain an upper bound we note first that $\varphi(x)-\varphi(x-t)>\varphi(x)$ and then use $1^{\circ}$ as follows:

$$
\begin{array}{r}
\varphi(x+t)-\varphi(x) \leq(\varphi(3 x)-\varphi(2 x))+(\varphi(2 x)-\varphi(x)) \\
\leq\left(\varrho_{1}+1\right)(\varphi(2 x)-\varphi(x)) \leq \varrho_{1}\left(\varrho_{1}+1\right) \varphi(x) .
\end{array}
$$


Hence $\psi(x, t) \leq \varrho_{1}\left(\varrho_{1}+1\right)$. If $x<t / 2$, we estimate as follows:

$\varphi(x)-\varphi(x-t)<\varphi\left(\frac{t}{2}\right)-\varphi(-t) \leq \varphi\left(\frac{t}{2}\right)+\varrho_{2} \varphi(t) \leq\left(1+\varrho_{2}\left(\varrho_{1}+1\right)\right) \varphi\left(\frac{t}{2}\right)$,

$\varphi(x+t)-\varphi(x)>\varphi(t)-\varphi\left(\frac{t}{2}\right) \geq \frac{1}{\varrho_{1}} \varphi\left(\frac{t}{2}\right)$,

$\varphi(x)-\varphi(x-t)>-\varphi\left(-\frac{t}{2}\right) \geq \frac{1}{\varrho_{2}} \varphi\left(\frac{t}{2}\right)$,

$\varphi(x+t)-\varphi(x)<\varphi(2 t) \leq\left(\varrho_{1}+1\right) \varphi(t) \leq\left(\varrho_{1}+1\right)^{2} \varphi\left(\frac{t}{2}\right)$.

From these inequalities we obtain

$$
\frac{1}{\varrho_{1}\left(1+\varrho_{2}\left(\varrho_{1}+1\right)\right)}<\psi(x, t)<\varrho_{2}\left(\varrho_{1}+1\right)^{2} .
$$

The equation (11) is thus satisfied with

$$
\begin{aligned}
\varrho & =\max \left\{\varrho_{1}\left(\varrho_{2}+1\right), \varrho_{1}\left(\varrho_{1}+1\right), \varrho_{1}\left(1+\varrho_{2}\left(\varrho_{1}+1\right)\right), \varrho_{2}\left(\varrho_{1}+1\right)^{2}\right\} \\
& =\varrho_{2}\left(\varrho_{1}+1\right)^{2} .
\end{aligned}
$$

In the case $x<0, x+t>0$, the equation (11) with the same $\varrho$ follows by symmetry. All the other cases are included in $1^{\circ}$ and $2^{\circ}$, and so the lemma is proved.

The following distortion lemma is a result of Agard and Gehring ([1], Theorem 2).

Lemma 6. Suppose that $f$ is a $K$-quasiconformal mapping of the extended plane and that $f(\infty)=\infty$. Then for each triple of distinct finite points $z_{1}, z_{2}, z_{3}$,

$$
\sin \frac{1}{2} \alpha \geq \mu^{-1}\left(K \mu\left(\sin \frac{1}{2} \beta\right)\right),
$$

where $\mu(r), 0<r<1$, is the modulus of the unit disk slit along the real axis from 0 to $r$, and

$$
\begin{aligned}
& \alpha=\arcsin \left(\lambda\left(z_{1}, z_{2}, z_{3}\right)\right)^{-1}, \\
& \beta=\arcsin \left(\lambda\left(f\left(z_{1}\right), f\left(z_{2}\right), f\left(z_{3}\right)\right)\right)^{-1},
\end{aligned}
$$

with $\lambda$ defined by the formula (4) in Section 3. This inequality is sharp.

Theorem 1. A closed Jordan arc $\gamma$ is quasiconformal if and only if it has bounded distortion.

Proof. Suppose first that the distortion $c=c(\gamma)$ of $\gamma$ is finite. We may assume that $\gamma$ has $\infty$ as one end-point, because linear transforma- 
tions affect neither the quasiconformality nor the distortion of $\gamma$. Let $\omega: C_{\gamma} \cup \Gamma \rightarrow \bar{H}$ be fixed as on p. 12 with $q=\infty$. We define a strictly increasing function $\varphi$ of the real line onto itself by setting

$$
\varphi(x)=\left\{\begin{array}{l}
x, \text { if } x>\vartheta, \\
\omega\left(z^{+}\right), \text {if }-\omega\left(z^{-}\right)=x>0 \text { and } z \in \gamma .
\end{array}\right.
$$

Hence $\varphi(0)=0$, and we will show that $\varphi$ satisfies also the other conditions of Lemma 5. Assume $t>0$ and $x-t \geq 0$, and let $z_{1}, z_{2}$, and $z_{3}$ be points of $\gamma$ such that $-\omega\left(z_{1}^{-}\right)=x-t,-\omega\left(z_{2}^{-}\right)=x$, and $-\omega\left(z_{3}^{-}\right)=x+t$. Then $M\left(C_{\gamma}\left(\infty, z_{3}^{-}, z_{2}^{-}, z_{1}^{-}\right)\right)=1$, and by Lemme 3 there exists a constant $k_{1}$ depending only on $c$ such that

$$
\frac{1}{k_{1}} \leq M\left(C_{\gamma}\left(z_{1}^{+}, z_{2}^{+}, z_{3}^{+}, \infty\right)\right) \leq k_{1} .
$$

But this implies $\left([5]\right.$, p. 84, 85) that there exists a $\varrho_{1}<\infty$ depending only on $k_{1}$ such that

$$
\frac{1}{\varrho_{1}} \leq \frac{\omega\left(z_{3}^{+}\right)-\omega\left(z_{2}^{+}\right)}{\omega\left(z_{2}^{+}\right)-\omega\left(z_{1}^{+}\right)} \leq \varrho_{1} .
$$

Since the case $x+t \leq 0$ is trivial, it follows from the definition of $\phi$ that the condition $1^{\circ}$ of Lemma 5 holds with constant $\varrho_{1}$. To prove $2^{\circ}$, let $z$ be a point of $\gamma$ such that $-\omega\left(z^{-}\right)=t>0$. Then it follows similarly from Lemma 4 that there exists a $\varrho_{2}<\infty$ depending only on $c$ such that

$$
\frac{1}{\varrho_{2}} \leq \frac{\omega\left(z^{+}\right)}{-\omega\left(z^{-}\right)} \leq \varrho_{2} \text {. }
$$

This implies $2^{\circ}$ by the definition of $\varphi$. Hence $\varphi$ is $\varrho$-quasisymmetric with $\varrho$ depending only on $c$. Then $\varphi^{-1}$ is also $\varrho$-quasisymmetric, and there exists a $K$-quasiconformal mapping $g: H \rightarrow H$ with $K$ depending only on $c$ such that $g$ induces the boundary correspondence $\varphi^{-1}$ ([3]). Let $h$ be the conformal mapping $z \rightarrow z^{2}$ of $H$ onto $C_{\overline{\mathbf{R}}_{+}}$, where $\mathbf{R}_{+}$is the positive real axis. The construction of the composed $K$-quasiconformal mapping $h \circ g \circ \omega: C_{\gamma} \rightarrow C_{\overline{\mathbf{R}}_{+}}$is carried out in such a way that it can be extended to a homeomorphism $f$ of the whole plane onto itself. But $f$ is then $K$-quasiconformal, because $\overline{\mathbf{R}}_{+}$is an analytic arc ([5], Satz I.8.3). Since $f(\gamma)=\overline{\mathbf{R}}_{+}$, the arc $\gamma$ is $K$-quasiconformal.

For the converse, suppose $\gamma$ is quasiconformal, and let $f$ be a $K$. quasiconformal mapping of the extended plane onto itself such that $f(\gamma)$ is a line segment. Let $z_{1}, z_{2}, z_{3}, z_{4}$ be a sequence of successive points of $\gamma$. and let $g$ and $h$ be linear transformations such that $g\left(z_{4}\right)=h\left(f\left(z_{4}\right)\right)=\infty$. 
Then $w=h \circ f \circ g^{-1}$ is a $K$-quasiconformal mapping of the extended plane keeping the point $\infty$ fixed. Since

$$
\lambda\left(h\left(f\left(z_{1}\right)\right), h\left(f\left(z_{2}\right)\right), h\left(f\left(z_{3}\right)\right)\right)=1
$$

we obtain, by applying Lemma 6 to the mapping $w$ and using known properties of $\mu$, the inequality

$$
\sin \frac{1}{2} \arcsin \left(\lambda\left(g\left(z_{1}\right), g\left(z_{2}\right), g\left(z_{3}\right)\right)\right)^{-1} \geq \mu^{-1}\left(K \mu\left(\frac{1}{\sqrt{2}}\right)\right)=\mu^{-1}\left(\frac{K \pi}{2}\right) .
$$

But

$$
\left|z_{1}, z_{4}, z_{2}, z_{3}\right|+\left|z_{1}, z_{2}, z_{4}, z_{3}\right|=\lambda\left(g\left(z_{1}\right), g\left(z_{2}\right), g\left(z_{3}\right)\right)
$$

(see p. 9), and so the distortion of $\gamma$ satisfies the inequality

$$
c(\gamma) \leq \frac{1}{\sin 2 \arcsin \mu^{-1}\left(\frac{K \pi}{2}\right)}
$$

The theorem is proved.

Remark. We point out that in the first part of the proof of Theorem 1 the mapping $f$ is $K$-quasiconformal, $K$ depending only on the distortion $c\left(\gamma^{\prime}\right)$.

4.3. Let $\bar{K}(\gamma)$ denote the infimum of the maximal dilatations $K(f)$, $f$ running over the set of the quasiconformal mappings of the extended plane which map $\gamma$ onto a line segment. According to the latter part of Lemma 6, the inequality (12) is sharp in the following sense: Given $K$, $1 \leq K<\infty$, there exists a closed Jordan arc $\gamma$ such that there is equality in (12) with $K=\bar{K}(\gamma)$. The left side of the following equivalent inequality

$$
\frac{2}{\pi} \mu\left(\sin \frac{1}{2} \arcsin \frac{1}{c(\gamma)}\right) \leq \bar{K}(\gamma)
$$

is thus a best possible lower bound for $\bar{K}(\gamma)$ in terms of $c(\gamma)$. A best possible lower bound for $K(\gamma)$ is obtained in terms of the maximal local distortion $c_{0}(\gamma)$ in the next section.

4.4. The known result corresponding to Theorem 1 for Jordan curves ([2]) follows here as a corollary.

Corollary. A Jordan curve $\gamma$ is quasiconformal if and only if it has bounded distortion.

Proof. Suppose that $c=c(\gamma)$ is finite. Let $\gamma_{1}, \gamma_{2}, \ldots$ be a sequence of closed subares of $\gamma$ such that $\gamma_{1} \subset \gamma_{2} \subset \ldots$ and that the end-points 
converge to a point of $\gamma$. Then $c\left(\gamma_{n}\right) \leq c, n=1,2, \ldots$, and there exist $K$-quasiconformal mappings $f_{n}$ of the extended plane, $K$ depending only on $c$, such that $f_{n}\left(\gamma_{n}\right)$ is a subarc of the unit circle. In addition, we can normalize the mappings $f_{n}$ so that they form a normal family. Then there exists a subsequence converging to a $K$-quasiconformal mapping $f$ of the whole plane. But $f(\gamma)$ is then the unit circle, and so $\gamma$ is $K$-quasiconformal. The converse statement can be proved exactly as in Theorem 1.

Clearly the inequality (13) holds and is sharp also in the case where $\gamma$ is a Jordan curve if $\bar{K}(\gamma)$ has the corresponding meaning.

\section{Characterization by local distortion}

5.1. The question arises whether the quasiconformality of an open Jordan arc can also be characterized by the distortion of the arc. The answer is negative, as is seen by the example on p. 11. For the arc (5) is quasiconformal as an analytic are, but it does not have bounded distortion. In the opposite direction we have, however, the following result.

Theorem 2. An open or a half-open Jordan arc with bounded distortion is $K$-quasiconformal, $K$ depending only on $c(\gamma)$.

Proof. Let $\gamma$ be an open Jordan arc with $c(\gamma)<\infty$. We show first that $\bar{\gamma}$ is a closed Jordan arc or a Jordan curve. Let $\varphi: I \rightarrow \gamma$ be a parameter representation for $\gamma$, where $I$ is the interval $(0,1)$. Take sequences $s_{1}, s_{2}, \ldots$ and $t_{1}, t_{2}, \ldots$ of points of $I$ such that $\lim _{n \rightarrow \infty} s_{n}=0$ and $\lim _{n \rightarrow \infty} t_{n}$ $=1$. Let $p$ and $q$ be limit points of the sets $\left\{\varphi\left(s_{1}\right), \varphi\left(s_{2}\right), \ldots\right\}$ and $\left\{\varphi\left(t_{1}\right), \varphi\left(t_{2}\right), \ldots\right\}$ respectively. Then $p$ and $q$ belong to the set $\bar{\gamma}-\gamma$. Suppose there exists a point $z \in \bar{\gamma}-\gamma$ such that $z \neq p, q$. Then there is a sequence $u_{1}, u_{2}, \ldots$ of points of $I$ such that one of the following conditions holds:

(a) $\lim _{n \rightarrow \infty} u_{n}=1$ and $z, q \in \overline{\left\{\varphi\left(u_{1}\right), \varphi\left(u_{2}\right), \ldots\right\}}$.

(b) $\lim _{n \rightarrow \infty} u_{n}=0$ and $z, p \in \overline{\left\{\varphi\left(u_{1}\right), \varphi\left(u_{2}\right), \ldots\right\}}$.

Suppose that (a) holds, and let $g$ be a linear transformation such that $g\left(\varphi\left(u_{0}\right)\right)=\infty$ for a point $u_{0} \in I$. Given $\varepsilon>0$, there exist points $u_{k}, u_{l}, u_{m} \in I$ such that $u_{0}<u_{k}<u_{l}<u_{m}$ and that $\left|g\left(\varphi\left(u_{k}\right)\right)-g(q)\right|$, $\left|g\left(\varphi\left(u_{l}\right)\right)-g(z)\right|,\left|g\left(\varphi\left(u_{m}\right)\right)-g(q)\right|<\varepsilon$. Hence $\left|g\left(\varphi\left(u_{k}\right)\right)-g\left(\varphi\left(u_{m}\right)\right)\right|<2 \varepsilon$ and $\left|g\left(\varphi\left(u_{k}\right)\right)-g\left(\varphi\left(u_{l}\right)\right)\right|>|g(q)-g(z)|-2 \varepsilon$. But this is in contradiction with the inequality $\left|g\left(\varphi\left(u_{k}\right)\right)-g\left(\varphi\left(u_{l}\right)\right)\right| \leq c(\gamma)\left|g\left(\varphi\left(u_{k}\right)\right)-g\left(\varphi\left(u_{m}\right)\right)\right|$ if $\varepsilon$ is sufficiently small. The assumption (b) leads similarly to a contradiction, and we have proved that $\bar{\gamma}-\gamma=\{p, q\}$. If $p \neq q, \bar{\gamma}$ is a 
closed Jordan arc, and in the case $p=q, \bar{\gamma}$ is a Jordan curve. It is easily verified that $c(\bar{\gamma})=c(\gamma)$, and so the assertion follows from Theorem 1 and its corollary and the remark on p. 19. The proof is similar for half-open arcs.

5.2. In order to obtain a characterization of quasiconformality also valid for open Jordan arcs, we shall use the concept of local distortion. We start by establishing the connection with local quasiconformality.

Theorem 3. A Jordan arc or curve $\gamma$ is quasiconformal at $z \in \gamma$ if and only if $c(\gamma, z)$ is finite. More precisely, the inequality

$$
\frac{2}{\pi} \mu\left(\sin \frac{1}{2} \arcsin \frac{1}{c(\gamma, z)}\right) \leq K(\gamma, z)
$$

is sharp, and there exists an upper bound for $K(\gamma, z)$ which depends only on $c(\gamma, z)$.

Proof. Let $c(\gamma, z)$ be finite. Then there exists a subarc $\gamma_{z} \ni z$ of $\gamma$ open in $\gamma$ and such that $c\left(\gamma_{z}\right)$ has an upper bound depending only on $c(\gamma, z)$. Hence, by Theorem 2, $\gamma_{z}$ is $K$-quasiconformal, $K$ depending only on $c(\gamma, z)$.

Assume that $\gamma$ is $K$-quasiconformal at $z \in \gamma$. Then there is a $K$ quasiconformal mapping $f$ of a domain $G \ni z$ such that $f(G \cap \gamma)$ is a segmert of the real line. It is no restriction to assume that $z=f(z)=0$. Let $U \subset G$ be a circular disk with centre 0 , and let $g$ map $f(U)$ conformally onto $U$ so that $g(0)=0$. Then $g \circ f$ maps $U$ onto itself $K$ quasiconformally, and reflecting in $\partial U^{\top}$ we obtain a $K$-quasiconformal mapping $h$ of the extended plane with $h(\infty)=\infty$. The set $h(U \cap \gamma)$ contains an analytic arc $\gamma_{0}^{\prime} \ni 0$ open in $h(U \cap \gamma)$. Since $\gamma_{0}^{\prime}$ has a continuous tangent, there exists for a given $\varepsilon>0$ a subarc $\gamma_{0} \ni 0$ of $\gamma_{0}^{\prime}$ open in $h(U \cap \gamma)$, such that for successive points $z_{1}, z_{2}, z_{3}$ of $\gamma_{0}$ the condition

$$
1-\varepsilon<\frac{1}{\lambda_{h}\left(h\left(z_{1}\right), h\left(z_{2}\right), h\left(z_{3}\right)\right)} \leq 1
$$

holds. Applying Lemma 6 to the mapping $h$ and using the properties of $\mu$, we obtain

$$
\lambda\left(z_{1}, z_{2}, z_{3}\right) \leq \frac{1}{\sin 2 \arcsin \mu^{-1}\left(\frac{K \pi}{2}\right)}+(\varepsilon)
$$

for successive points $z_{1}, z_{2}, z_{3}$ of $\gamma_{0}$, where $(\varepsilon) \rightarrow 0$, as $\varepsilon \rightarrow 0$. From this it follows that $c(\gamma, z)$ is finite and that the inequality (14) holds. 
To prove that (14) is best possible, we make use of the connection with the inner measure of topological angles. Given $\varepsilon>0,0<\alpha \leq \pi$, and $1 \leq K<\infty$, there exists by [1], Theorem 3, a $K$-quasiconformal mapping $f$ of a domain $G$ and two line segments $\gamma_{1}$ and $\gamma_{2}$ which form an angle in $G$, such that $A\left(\gamma_{1}, \gamma_{2}\right)=\alpha$ and

$$
\sin \frac{1}{4} A\left(f\left(\gamma_{1}\right), f\left(\gamma_{2}\right)\right) \leq \mu^{-1}\left(K \mu\left(\sin \frac{1}{4} A\left(\gamma_{1}, \gamma_{2}\right)\right)\right)+\varepsilon .
$$

We apply this to the case $\alpha=\pi$, i.e. when $\gamma_{1} \cup \gamma_{2}$ is a line segment. Let $\gamma$ be the Jordan arc $f\left(\gamma_{1}\right) \cup f\left(\gamma_{2}\right)$ and $z$ the common point of $f\left(\gamma_{1}\right)$ and $f\left(\gamma_{2}\right)$. Then by $(6)$ and (15) we obtain

$$
\sin \frac{1}{2} \arcsin \frac{1}{c(\gamma, z)} \leq \sin \frac{1}{4} A\left(f\left(\gamma_{1}\right), f\left(\gamma_{2}\right)\right) \leq \mu^{-1}\left(\frac{K \pi}{2}\right)+\varepsilon,
$$

and so

$$
\frac{2}{\pi} \mu\left(\sin \frac{1}{2} \arcsin \frac{1}{c(\gamma, z)}\right)+(\varepsilon) \geq K,
$$

where $(\varepsilon) \rightarrow 0$, as $\varepsilon \rightarrow 0$. This proves the statement.

Remark. If $z \in \gamma$ is not an end-point of $\gamma$, we obtain an upper bound for $K(\gamma, z)$ in terms of $c(\gamma, z)$ also by enclosing a subare of $\gamma$, which contains the point $z$, to a Jordan curve as follows: We may assume that $z=0$. Let $U=\{z|| z \mid<r\}$ be a disk such that there exist points $z^{\prime}, z^{\prime \prime} \in \gamma \cap \partial \mathrm{U}$ with $0 \in \gamma\left(z^{\prime}, z^{\prime \prime}\right) \subset U$. We can choose $U$ so that the subarc $\gamma_{U}=\gamma\left(z^{\prime}, z^{\prime \prime}\right)$ satisfies the inequality

$$
\sup _{z_{2} \in \gamma_{\left.U^{\left(z_{1},\right.}, z_{3}\right)}} \lambda\left(z_{1}, z_{2}, z_{3}\right) \leq 2 c(\gamma, 0) \text {. }
$$

It is not difficult to show that the Jordan curve $J_{0}=\gamma_{U} \cup L^{\prime} \cup L^{\prime \prime} \cup\{\infty\}$, where $L^{\prime}=\left\{z\left|\arg z=\arg z^{\prime},\right| z \mid \geq r\right\} \quad$ and $L^{\prime \prime}=\left\{z \mid \arg z=\arg z^{\prime \prime}\right.$, $|z| \geq r\}$, has bounded distortion with $c\left(J_{0}\right)$ depending only on $c(\gamma, 0)$. Then the result of Ahlfors [2] says that $J_{0}$ is $K$-quasiconformal, $K$ depending only on $c(\gamma, 0)$. Hence $K(\gamma, 0)$ has an upper bound depending only on $c(\gamma, 0)$.

Directly from the definitions and Theorem 3 we conclude that the corresponding inequality

$$
\frac{2}{\pi} \mu\left(\sin \frac{1}{2} \arcsin \frac{1}{c_{0}(\gamma)}\right) \leq K(\gamma)
$$

holds and is best possible. 
5.3. We shall now show that a Jordan arc or curve $\gamma$ with bounded local distortion is quasiconformal. Moreover, there exists an upper bound for $K(\gamma)$ depending only on $c_{0}(\gamma)$. The former result follows for closed Jordan arcs and Jordan curves from Lemma 2, Theorem 1, and its corollary. We first prove some preliminary results.

Lemma 7. Let $\gamma$ be an open Jordan arc. Then there exists a simply connected domain which has $\gamma$ as a free boundary arc.

Proof. According to our definition of a free boundary arc of a domain, we have to construct a simply connected domain $D$ which has $\gamma$ as a boundary arc such that $\overline{\partial D-\gamma} \cap \gamma=\varnothing$ and that $\partial D-\gamma$ is connected.

Fix a natural ordering $<$ on the points of $\gamma$. Let $z_{n}$ and $w_{n}$ be points of $\gamma$ such that $z_{n+1}<z_{n}<\ldots<z_{1}<w_{1}<\ldots<w_{n}<w_{n+1}$, $n=1,2, \ldots$, and that the arcs $\gamma\left(z_{n}, w_{n}\right)$ cover $\gamma$. Denote $\gamma_{n}^{\prime}=\{z \in \gamma \mid$ $\left.z<z_{n}\right\}$ and $\gamma_{n}^{\prime \prime}=\left\{z \in \gamma \mid z>w_{n}\right\}, n=1,2, \ldots$

Suppose that we have constructed Jordan domains $D_{k}$ and $E_{k}$, $k=1, \ldots, n$, such that $D_{k} \subset D_{k+1}, E_{k} \subset E_{k+1}, k=1, \ldots, n-1$, and $\bar{D}_{k} \cap \bar{E}_{k}=\bar{D}_{k} \cap \bar{\gamma}=\bar{E}_{k} \cap \bar{\gamma}=\overline{\gamma\left(z_{k}, w_{k}\right)}, \quad k=1, \ldots, n$. Let now $l_{n+1}$ be a Jordan arc with end-points $z_{n+2}$ and $w_{n+2}$ such that $J_{n+1}=l_{n+1}$ $\cup \gamma\left(z_{n+2}, w_{n+2}\right)$ is a Jordan curve and that $l_{n+1} \cap\left(\bar{D}_{n} \cup \bar{E}_{n}\right)=\varnothing$. Denote $\alpha_{n}=\partial D_{n}-\overline{\gamma\left(z_{n}, w_{n}\right)}, \quad \beta_{n}=\partial E_{n}-\overline{\gamma\left(z_{n}, w_{n}\right)}, \quad$ and $\quad \gamma_{\alpha_{n}}=\gamma_{n}^{\prime} \cup \bar{\alpha}_{n} \cup \gamma_{n}^{\prime \prime}$, $\gamma_{\beta_{n}}=\gamma_{n}^{\prime} \cup \bar{\beta}_{n} \cup \gamma_{n}^{\prime \prime}$. There is a Jordan domain $U_{n+1}$ which contains the arc $\overline{\gamma_{\alpha_{n}}\left(z_{n+1}, w_{n+1}\right)}$ and for which $\left.U_{n+1} \cap \overline{\left(\gamma_{n+2}^{\prime}\right.} \cup \overline{\gamma_{n+2}^{\prime \prime}} \cup l_{n+1}\right)=\varnothing$. Let $A_{n+1}$ be the complementary domain of $J_{n+1}$ which does not intersect $D_{n} \cup E_{n}$. By an argument similar to the one in the proof of Theorem VI.14.6 in [6], it is shown that there exists an open Jordan arc $\alpha_{n+1} \subset A_{n+1} \cap U_{n+1}$ with end-points $z_{n+1}$ and $w_{n+1}$. A similar construction applied to $\gamma_{\beta_{n}}$ yields an arc $\beta_{n+1}$ (Fig. 1). Then $\bar{\alpha}_{n+1} \cup \gamma\left(z_{n+1}, w_{n+1}\right)$ and $\bar{\beta}_{n+1} \cup \gamma\left(z_{n+1}, w_{n+1}\right)$ bound Jordan domains $D_{n+1}$ and $E_{n+1}$ with the properties $D_{n} \subset D_{n+1}, E_{n} \subset$ $E_{n+1}, \bar{D}_{n+1} \cap \bar{E}_{n+1}=\bar{D}_{n+1} \cap \bar{\gamma}=\bar{E}_{n+1} \cap \bar{\gamma}=\overline{\gamma\left(z_{n+1}, w_{n+1}\right)}$. If we set $D_{0}=E_{0}=\varnothing$, we have constructed sequences $D_{1}, D_{2}, \ldots$ and $E_{1}, E_{2}, \ldots$ of Jordan domains with the properties stated above. We assert that the simply connected domain

$$
D=\bigcup_{n=1}^{\infty} D_{n}
$$

has the required property in the lemma.

Clearly $\gamma \subset \partial D$. If $z \in \gamma$, there exists a neighbourhood $U$ of $z$ which is contained in $D_{k} \cup \gamma\left(z_{k}, w_{k}\right) \cup E_{k}$ for some $k$. By the construction of the sets $D_{n}$ it follows that $U \cap(\partial D-\gamma)=\varnothing$. Hence $\overline{\partial D-\gamma} \cap \gamma=\varnothing$. 


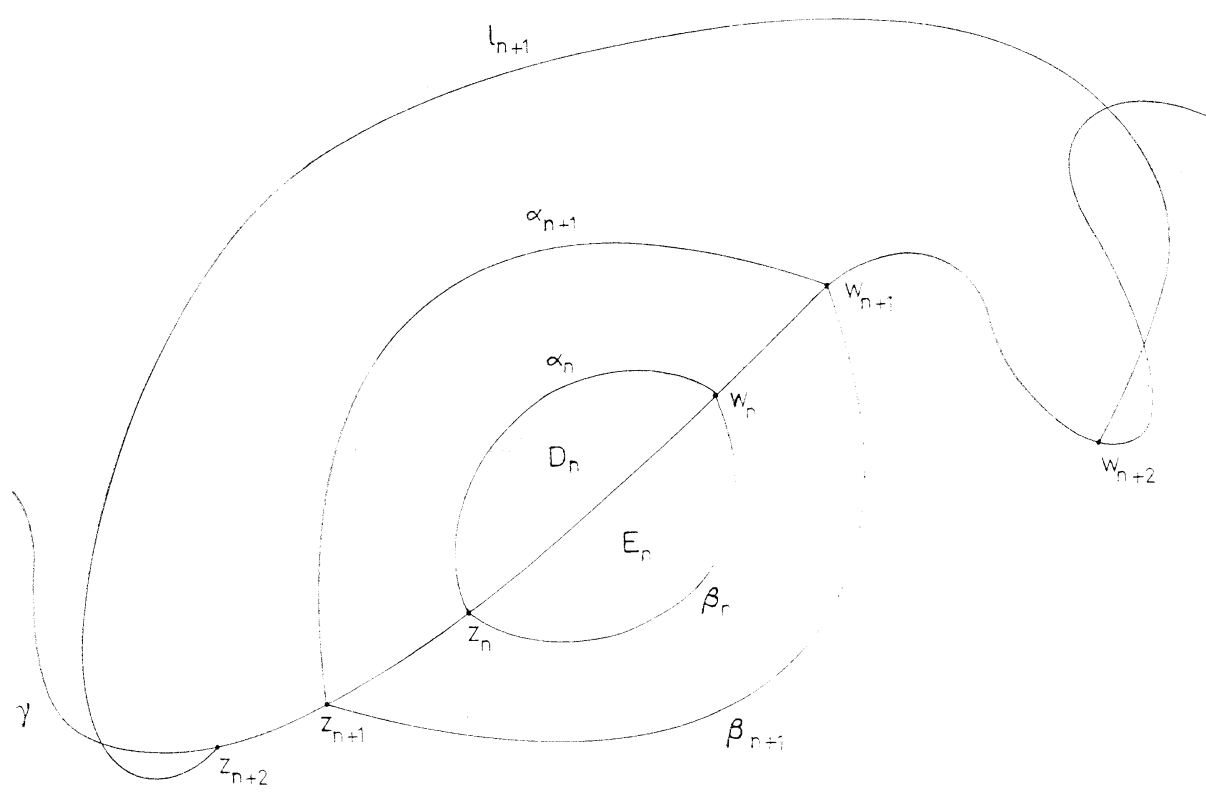

Figure 1.

It remains to be shown that $\partial D-\gamma$ is connected. We prove first that $L^{\prime}=\bar{\gamma}_{1}^{\prime}-\gamma$ and $L^{\prime \prime}=\bar{\gamma}_{1}^{\prime \prime}-\gamma$ are connected. Suppose that $\bar{\gamma}_{1}^{\prime}-\gamma=$ $A \cup B$, where $A$ and $B$ are non-empty, disjoint, and closed in $\bar{\gamma}_{1}^{\prime}-\gamma$. Since $\bar{\gamma}_{1}^{\prime}-\gamma$ is closed, the sets $A$ and $B$ are closed. Hence there exist disjoint neighbourhoods $M \supset A$ and $N \supset B$. There is a sequence $a_{1}, a_{2}, \ldots$ of successive points of $\gamma_{1}^{\prime}$ such that $a_{2 k-1} \in M$ and $a_{2 k} \in N$, $k=1,2, \ldots$. Every subarc $\gamma\left(a_{2 k-1}, a_{2 k}\right)$ has a point $b_{k} \notin M \cup N$, and the set $\left\{b_{1}, b_{2}, \ldots\right\}$ has a limit point $b \notin A \cup B$. But $b \in \bar{\gamma}_{1}^{\prime}-\gamma$, and so we have a contradiction. Hence $L^{\prime}$ is connected. The proof for $L^{\prime \prime}$ is similar.

Assume that $\partial D-\gamma=S \cup T$, where $S$ and $T$ are non-empty, disjoint, and closed. Let $V \supset S$ and $W \supset T$ be disjoint and open. Since $\gamma \subset \partial D$, we have $L^{\prime} \cup L^{\prime \prime}=\bar{\gamma}-\gamma \subset \partial D-\gamma$. Hence, in view of connectedness, $L^{\prime}$ is contained either in $S$ or in $T$. The same holds for $L^{\prime \prime}$. It suffices to consider the cases $L^{\prime}, L^{\prime \prime} \subset S$ and $L^{\prime} \subset S, L^{\prime \prime} \subset T$. Assume $L^{\prime}, L^{\prime \prime} \subset S$. Then $T \cap \bar{\gamma}=\varnothing$. Take a point $z \in T$ and a circular disk $U \subset W$ with centre $z$ such that $\bar{\gamma} \cap U=\varnothing$. Since $z \in \partial D$, there exists a point $d_{k} \in D_{k} \cap U$ for some $k$. From the monotonicity of the sequence $D_{1}, D_{2}, \ldots$ it follows that $d_{k} \in D_{n}$ for $n \geq k$. But then there exists a point $c_{n} \in \partial D_{n} \cap U$ for every $n \geq k$, because $z \notin D_{n}, n=1,2, \ldots$. This implies $c_{n} \in \alpha_{n}$ for $n \geq k$. On the other haıid, there is an $m$ such that the end-points $z_{n}$ of the arcs $\alpha_{n}$ belong to $V$ for $n \geq m$. Hence 
there exists a point $e_{n} \in \alpha_{n}-(V \cup W)$ for every $n \geq \max \{k, m\}$. Let $e$ be a limit point of the points $e_{n}$. Then $e$ is a point of $\partial D$ which does not belong to $S \cup T$. But $e \notin \gamma$, and so we have a contradiction. The case $L^{\prime} \subset S, L^{\prime \prime} \subset T$ leads similarly to a contradiction if we consider endpoints of the arcs $\alpha_{n}$. Hence $\partial D-\gamma$ is connected and the lemma is proved.

Lemma 8. Let $G$ and $G^{\prime}$ be domains with free boundary arcs or curves $\gamma$ and $\gamma^{\prime}$ respectively. Let $\gamma_{i}$ and $\gamma_{i}^{\prime}, i=1,2, \ldots$, be open subarcs of $\gamma$ and $\gamma^{\prime}$ satisfying the following conditions:

(a) $\gamma_{i} \cap \gamma_{j}=\gamma_{i}^{\prime} \cap \gamma_{j}^{\prime}=\varnothing$, if $i \neq j$.

(b) $c\left(\gamma_{i}\right) \leq c<\infty, c\left(\gamma_{i}^{\prime}\right) \leq c^{\prime}<\infty, i=1,2, \ldots$

If $f: G \rightarrow G^{\prime}$ is a K-quasiconformal mapping, where $\gamma_{i}$ and $\gamma_{i}^{\prime}$ correspond to each other, then there exists an extension of $f$ to a $\hat{K}$-quasiconformal mapping $g$ of a domain $\hat{G} \supset G \cup \bigcup_{i=1}^{\infty} \gamma_{i}, \hat{K}$ depending only on $K, c$, and $c^{\prime}$.

Proof. By Theorem 2, $\gamma_{i}$ and $\gamma_{i}^{\prime}$ are $K_{c^{-}}$and $K_{c^{\prime}}$-quasiconformal, where $K_{c}$ and $K_{c^{\prime}}$ depend only on $c$ and $c^{\prime}$. From Lemma 1 it follows that $f$ can be extended to $\hat{K}=K_{c}^{2} K K_{c^{\prime}}^{2}$-quasiconformal mappings $g_{i}$ of domains $G_{i} \supset G \cup \gamma_{i}, i=1,2, \ldots$ To complete the proof we restrict the mappings $g_{i}$ as follows. There exist open sets $U_{i} \supset \gamma_{i}, i=1,2, \ldots$, such that $U_{i} \subset G_{i}$ and $\bar{U}_{i} \cap \bigcup_{j \neq i} \gamma_{j}=\overline{g_{i}\left(U_{i}\right)} \cap \bigcup_{j \neq i} \gamma_{j}^{\prime}=\varnothing$. We define

$$
S_{i}=U_{i}-\bigcup_{k=1}^{i-1} \bar{U}_{k} \cup g_{i}^{-1}\left(\bigcup_{k=1}^{i-1} \overline{g_{k}\left(U_{k}\right)}\right) \text {. }
$$

Then every set $S_{i}$ is open and contains $\gamma_{i}$. Moreover, $S_{i} \cap S_{j}=$ $g_{i}\left(S_{i}\right) \cap g_{j}\left(S_{j}\right)=\varnothing$, if $i \neq j$. Let $T_{i}$ be the component of $S_{i}$ which contains the arc $\gamma_{i}$. We obtain the required $\hat{K}$-quasiconformal extension $g$ of $f$ defined in the domain

$$
\hat{G}=G \cup \bigcup_{i=1}^{\infty} T_{i} \supset G \cup \bigcup_{i=1}^{\infty} \gamma_{i}
$$

by setting

$$
g(z)=\left\{\begin{array}{l}
f(z), \text { if } z \in G, \\
g_{i}(z), \text { if } z \in T_{i} .
\end{array}\right.
$$

5.4. If the distortion $c(\gamma)$ of a Jordan arc or curve $\gamma$ is 1 , then $\gamma$ is contained in a line or a circle. Accordingly, in this case $K(\gamma)=1$. The question arises whether $c_{0}(\gamma)=1$ also implies $K(\gamma)=1$. One result in this direction will be presented in Theorem 4, namely, if $\gamma$ has a continuous 
tangent (in which case $c_{0}(\gamma)=1$ ), then $K(\gamma)=1$. We point out that examples can be given which show that $c_{0}(\gamma)=1$ does not imply the existence of the tangent.

Theorem 4. A Jordan arc or curve $\gamma$ is quasiconformal if and only if $c_{0}(\gamma)$ is finite. There exists an upper bound for $K(\gamma)$ depending only on $c_{0}(\gamma)$. Especially, if $\gamma$ has a continuous tangent, then $K(\gamma)=1$.

Proof. If $\gamma$ is quasiconformal, the inequality (16) shows that $c_{0}(\gamma)$ is finite.

Let us assume, conversely, that $c_{0}(\gamma)$ is finite. We consider first the case where $\gamma$ is an open Jordan arc. By Lemma 7 there exists a simply connected domain $G$ which has $\gamma$ as a free boundary arc. It is no restriction to assume that $\infty \in G$. Let $f$ map $G$ onto the upper half plane $H$ conformally so that $\gamma$ corresponds to a segment of the real axis $\mathbf{R}$. Every point $z \in \gamma$ is contained in an open subarc $\gamma_{z} \subset \gamma$ for which

$$
\sup _{z_{2} \in \gamma_{z}\left(z_{1}, z_{3}\right)} \lambda\left(z_{1}, z_{2}, z_{3}\right) \leq 2 c_{0}(\gamma)
$$

We cover $\gamma$ by a countable set of such subarcs $\gamma_{z}$ and denote these by $\gamma_{i}, i \in \mathbf{Z}$, where $\mathbf{Z}$ is the set of the integers. We can assume that $\gamma_{i} \cap \gamma_{i+1} \neq \varnothing, i \in \mathbf{Z}$, and $\gamma_{i} \cap \gamma_{j}=\varnothing$, if $i-j \geq 2$. Then the indices correspond to the successive order of the arcs (see Fig. 2). Let $\alpha_{i} \subset \gamma_{i}$ be disjoint open subarcs such that $\alpha_{i} \cap \gamma_{i-1} \neq \varnothing, i \in \mathbf{Z}$. The inequality (17) implies that the distortions $c\left(\alpha_{i}\right)$ of $\alpha_{i}, i \in \mathbf{Z}$, have an upper bound depending only on $c_{0}(\gamma)$. Hence there exists by Lemma 8 a $K$-quasiconformal extension $g$ of $f$ defined in a domain $D \supset G \cup \cup_{i \in \mathbf{Z}} \alpha_{i}, K$ depending only on $c_{0}(\gamma)$.

Let $z_{i}^{\prime}$ and $z_{i}^{\prime \prime}$ be two distinct points of $\alpha_{i}$ such that $z_{i}^{\prime \prime} \in \gamma_{i \perp 1}$ and that $z_{i-1}^{\prime}, z_{i-1}^{\prime \prime}, z_{i}^{\prime}$ are successive for every $i \in \mathbf{Z}$. Denote $w_{i}^{\prime}=g\left(z_{i}^{\prime}\right)$ and $w_{i}^{\prime \prime}=g\left(z_{i}^{\prime \prime}\right)$. We may assume $w_{i}^{\prime}<w_{i}^{\prime \prime}, i \in \mathbf{Z}$. Iet $u$ be a function of $\mathbf{R}$, which is negative in the intervals $\left(w_{i}^{\prime}, w_{i}^{\prime \prime}\right)$ and 0 elsewhere, and which has a continuous derivative with $u^{\prime}\left(w_{i}^{\prime}\right)=u^{\prime}\left(w_{i}^{\prime \prime}\right)=0, \quad i \in \mathbf{Z}$. Denote by $L$ the Jordan arc which has a parameter representation $\varphi: \mathbf{R} \rightarrow L$ with $\varphi(x)=x+\mathrm{i} u(x)$. In addition, we can choose $u$ so that the domain $A_{i} \nexists \infty$ bounded by the Jordan curve $L\left(w_{i}^{\prime}, w_{i}^{\prime \prime}\right) \cup$ $\left[w_{i}^{\prime}, w_{i}^{\prime \prime}\right]$ is contained in $g(D)$ for every $i \in \mathbf{Z}$. By an argument similar to the one used in the proof of Theorem 3 , it is shown that there exist disjoint open subares $l_{i}^{\prime} \ni z_{i}^{\prime}$ and $l_{i}^{\prime \prime} \ni z_{i}^{\prime \prime}, i \in \mathbf{Z}$, of $l=g^{-1}(L)$ such that

$$
\lambda\left(z_{1}, z_{2}, z_{3}\right) \leq \frac{1}{\sin 2 \arcsin \mu^{-1}\left(\frac{K \pi}{2}\right)}+1
$$




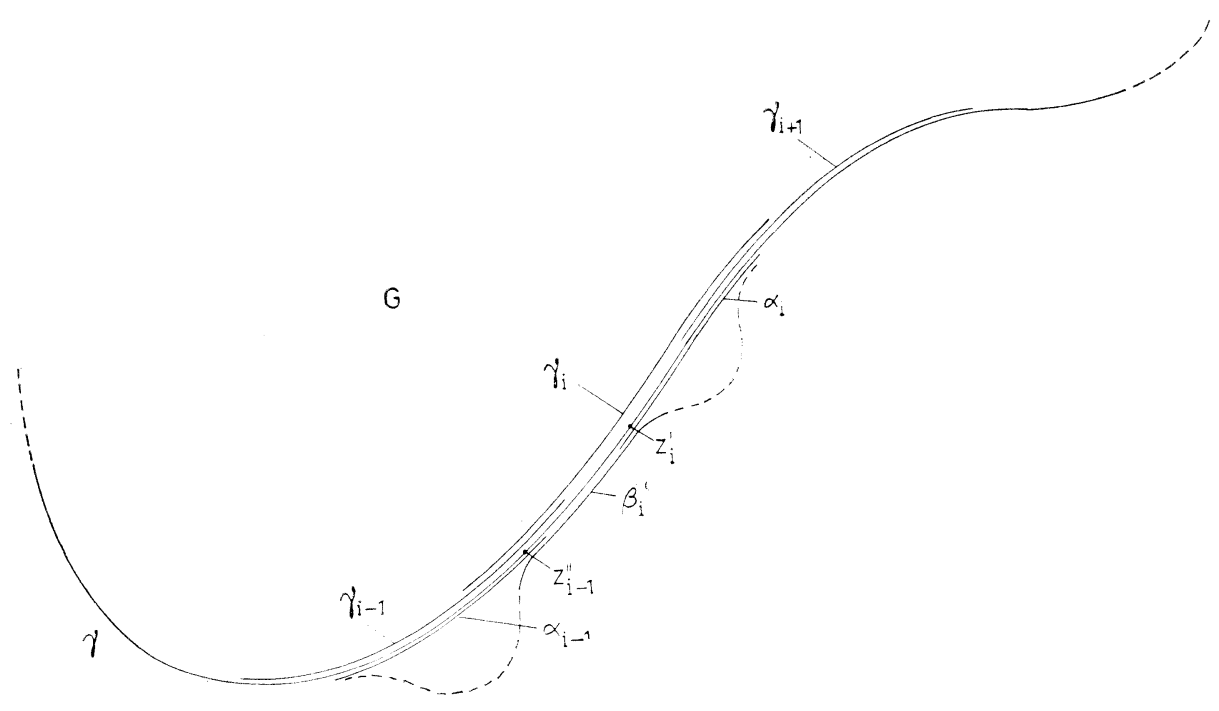

Figure 2.

for successive points $z_{1}, z_{2}, z_{3}$ of these arcs. Denote

$$
r_{i}=\min \left\{d\left(z_{i-1}^{\prime \prime},\left(\gamma_{i}^{\prime \prime \prime} \cup l_{i}^{\prime}\right)-l_{i-1}^{\prime \prime}\right), d\left(z_{i}^{\prime},\left(\gamma_{i}^{\prime \prime \prime} \cup l_{i-1}^{\prime \prime}\right)-l_{i}^{\prime}\right)\right\},
$$

where $\gamma_{i}^{\prime \prime \prime}=\gamma\left(z_{i-1}^{\prime \prime}, z_{i}^{\prime}\right), i \in \mathbf{Z}$. Let $\gamma_{i-1}^{\prime \prime} \ni z_{i-1}^{\prime \prime}$ and $\gamma_{i}^{\prime} \ni z_{i}^{\prime}$ be open subarcs of $l_{i-1}^{\prime \prime}$ and $l_{i}^{\prime}$ respectively with diameters less that $\varrho_{i}=r_{i} / 3$.

We shall show that the distortions of the arcs $\beta_{i}=\gamma_{i-1}^{\prime \prime} \cup \overline{\gamma_{i}^{\prime \prime \prime}} \cup \gamma_{i}^{\prime}$, $i \in \mathbf{Z}$, have an upper bound depending only on $c_{0}(\gamma)$. Let $z_{1}, z_{2}, z_{3}$ be successive points of $\beta_{i}$. If $z_{1}, z_{3} \in \gamma_{i}^{\prime \prime \prime}$, we have $\lambda\left(z_{1}, z_{2}, z_{3}\right) \leq 2 c_{0}(\gamma)$ in view of the choice of the arcs $\gamma_{i}$. If $z_{1}, z_{3} \in l_{i-1}^{\prime \prime}$ or $z_{1}, z_{3} \in l_{i}^{\prime}$, the inequality (18) gives an upper bound for $\lambda\left(z_{1}, z_{2}, z_{3}\right)$. To complete the proof of the assertion, it suffices, for reasons of symmetry, to consider the following three cases:

(a) $z_{1} \notin l_{i}^{\prime}$ and $z_{2}, z_{3} \in \gamma_{i}^{\prime}$.

(b) $z_{1} \in \gamma_{i-1}^{\prime \prime}, z_{2} \notin \gamma_{i-1}^{\prime \prime} \cup \gamma_{i}^{\prime}$, and $z_{3} \in \gamma_{i}^{\prime}$.

(c) $z_{1} \notin \gamma_{i-1}^{\prime \prime} \cup l_{i}^{\prime}, z_{2} \notin \gamma_{i}^{\prime}$, and $z_{3} \in \gamma_{i}^{\prime}$.

In the case (a) we have the estimates $\left|z_{1}-z_{2}\right| \leq\left|z_{1}-z_{i}^{\prime}\right|+\varrho_{i},\left|z_{2}-z_{3}\right|$ $\leq \varrho_{i}$, and $\left|z_{1}-z_{3}\right| \geq\left|z_{1}-z_{i}^{\prime}\right|-\varrho_{i}$. An upper bound for $\lambda\left(z_{1}, z_{2}, z_{3}\right)$ is then obtained as follows:

$$
\lambda\left(z_{1}, z_{2}, z_{3}\right) \leq \frac{\left|z_{1}-z_{i}^{\prime}\right|+2 \varrho_{i}}{\left|z_{1}-z_{i}^{\prime}\right|-\varrho_{i}} \leq \frac{1+2 / 3}{1-1 / 3} .
$$


In the case (b) we have

$$
\lambda\left(z_{1}, z_{2}, z_{3}\right) \leq \frac{\left|z_{i-1}^{\prime \prime}-z_{2}+\varrho_{i}+\right| z_{2}-z_{i}^{\prime} \mid+\varrho_{i}}{\left|z_{i-1}^{\prime \prime}-z_{i}^{\prime}\right|-2 \varrho_{i}} \leq 2 c_{0}(\gamma) \frac{1+2 / 3}{1-2 / 3},
$$

and in the case $(c)$

$$
\lambda\left(z_{1}, z_{2}, z_{3}\right) \leq \frac{\left|z_{1}-z_{2}+\right| z_{2}-z_{i}^{\prime}+\varrho_{i}}{\mid z_{1}-z_{i}^{\prime}-\varrho_{i}} \leq 2 c_{0}(\gamma) \frac{1+1 / 3}{1-1 / 3} .
$$

In addition, the ares $\gamma_{i-1}^{\prime \prime}$ and $\gamma_{i}^{\prime}$ can be chosen so that $c\left(g\left(\beta_{i}\right)\right) \leq 2$, $i \in \mathbf{Z}$. Applying then Lemma 8 to the arcs $\beta_{i}, i \in \mathbf{Z}$, and to the mapping $g$ restricted to the domain $G \cup \underset{i \in \mathbf{Z}}{\cup}\left(\gamma\left(z_{i}^{\prime}, z_{i}^{\prime \prime}\right) \cup g^{-1}\left(A_{i}\right)\right)$, we obtain a $\hat{K}$ quasiconformal mapping $h$ of a domain $\hat{G} \supset \gamma$, which maps $\gamma$ onto a segment of the real line, and where $\hat{K}$ depends only on $c_{0}(\gamma)$. We have thus proved that $K(\gamma)$ has an upper bound depending only on $c_{0}(\gamma)$, if $\gamma$ is an open Jordan arc.

In case $\gamma$ is a Jordan curve the proof is easier, because Lemma 7 is not needed and the covering of $\gamma$ by the arcs $\gamma_{i}$ can be chosen to be finite. Let $\gamma$ be a closed Jordan arc. If we apply Theorem 3 to the end-points of $\gamma$, it follows that $\gamma$ is contained in an open Jordan arc $\gamma_{0}$ such that $c_{0}\left(\gamma_{0}\right)$ has an upper bound depending only on $c_{0}(\gamma)$. The same holds clearly for a half-open arc.

To prove the last statement in the theorem, suppose that $\gamma$ has a continuous tangent. Assume that $\gamma$ is an open Jordan arc. Let $z_{0}$ be a point of $\gamma$. By performing a linear transformation, we may assume that $\gamma$ has a horizontal tangent at $z_{0}$ and that $z_{0}=0$. Then there exists an open subarc $\gamma_{*} \ni 0$ of $\gamma$ which has the parameter representation $\psi:(-\delta, \delta) \rightarrow \gamma_{*}$ defined by $\psi(x)=x+\mathrm{i} v(x)$, where $v$ is continuously differentiable, $v^{\prime}(0)=0$, and $v^{\prime}(x)<1$ for $x \in(-\delta, \delta)$. We define a mapping $w$ of the strip domain $\{z|| \operatorname{Re} z \mid<\delta\}$ onto itself by $w(x+\mathrm{i} y)$ $=x+\mathrm{i}(y+v(x))$. Then $w$ maps the interval $(-\delta, \delta)$ onto the arc $\gamma_{*}$, and the dilatation quotient $D(z)$ of $w$ at $z=x+\mathrm{i} y$ ([5], p. 18) satisfies the inequality

$$
D(z) \leq \frac{1+v^{\prime}(x) \mid}{1-\left|v^{\prime}(x)\right|}
$$

Given $\varepsilon>0$, we cover $\gamma$ by arcs $\gamma_{i}, i \in \mathbf{Z}$, as above so that the oscillation $t\left(\gamma_{i}\right)$ of the direction of the tangent of $\gamma_{i}$ is less than $\varepsilon$ for every $i \in \mathbf{Z}$. Let $f$ and $\alpha_{i}, i \in \mathbf{Z}$, be as before. The extension $g$ of $f$ is now constructed by the use of mappings like $w$ (cf. the proofs of Lemma 1 $([5]$, p. 102) and Lemma 8). Then (19) shows that $g$ is $1+(\varepsilon)$-quasicon- 
formal, where $(\varepsilon) \rightarrow 0$, as $\varepsilon \rightarrow 0$. We can here choose the arcs $\beta_{i}$ so that the corresponding oscillations $t\left(\beta_{i}\right)$ and $t\left(g\left(\beta_{i}\right)\right)$ are less than $2 \varepsilon$. If we apply the same method to the arcs $\beta_{i}$ and $g\left(\beta_{i}\right)$, we can also construct the extension $h$ so that it is $1+(\varepsilon)$-quasiconformal, where $(\varepsilon) \rightarrow 0$, as $\varepsilon \rightarrow 0$. Hence $K(\gamma)=1$. In case $\gamma$ is a Jordan curve, we use again a corresponding finite covering of $\gamma$, and in case $\gamma$ is a closed or a halfopen Jordan arc, we have only to remark that $\gamma$ is contained in an open Jordan arc with a continuous tangent. The theorem is proved.

5.5. Combining Theorems 3 and 4, we obtain the following result.

Corollary. Let $\gamma$ be a closed Jordan arc or a Jordan curve. Then $\gamma$ is quasiconformal if and only if it is quasiconformal at every point $z \in \gamma$.

Note that this follows also by Lemma 2, Theorem 3, Theorem 1 and its corollary.

The corresponding statement for open Jordan arcs does not hold. This is shown by the example in Figure 3, where $x_{n}=1 / n, n=1,2, \ldots$.

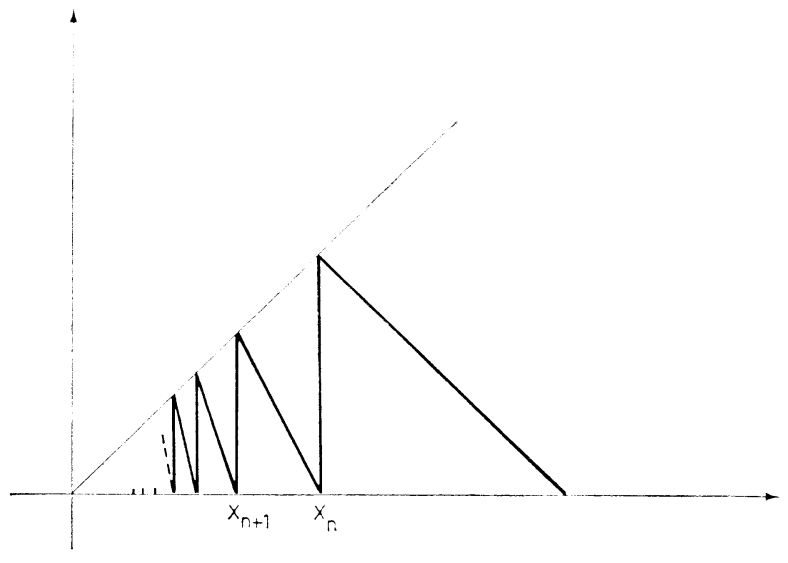

Figure 3.

University of Helsinki

Helsinki, Finland 


\section{References}

[1] Agard, S. B. - Gehring, F. W.: Angles and quasiconformal mappings. - Proc. London Math. Soc. 3. 14 A (1965), 1-21.

[2] Ahlfors, L.: Quasiconformal reflections. - Acta Math. 109 (1963), 291-301.

[3] Beurling, A. - Ahlfors, L.: The boundary correspondence under quasiconformal mappings. - Acta Math. 96 (1956), 125-142.

[4] Gehring, F. W.: Extension of quasiconformal mappings in three space. - J. Analyse Math. 14 (1965), 171-182.

[5] Lehto, O. - Virtanes, K. I.: Quasikonforme Abbildungen. - Springer-Verlag, 1965.

[6] Newman, M. H. A.: Elements of the topology of plane sets of points. - Cambridge University Press, 1964.

[7] Pfluger, A.: Über die Konstruktion Riemannscher Flächen durch Verheftung. - J. Indian Math. Soc. 24 (1960), 401-412.

[8] Tienari, M.: Fortsetzung einer quasikonformen Abbildung über einen Jordanbogen. - Ann. Acad. Sci. Fenn. A I 321 (1962).

[9] VärsäLÄ, J.: Remarks on a paper of Tienari concerning quasiconformal continuation. - Ann. Acad. Sci. Fenn. A I 324 (1962). 